\title{
Les défis structurels, organisationnels et cognitifs liés à la gestion de I'hétéropgénéité des élèves dans les systèmes éducatifs
}

\section{Farah Dubois-Shaik et Vincent Dupriez}

Ce texte s'intéresse aux différentes voies d'identification et de traitement de la question de l'hétérogénéité des élèves dans les systèmes éducatifs. Il convoque des résultats de recherche bien établis faisant apparaître combien le regroupement d'élèves vulnérables dans des groupes spécifiques (qu'il s'agisse de classes, de filières, d'options...) s'accompagne presque systématiquement d'un accroissement des différences entre ces élèves et les autres, à travers notamment un processus d'adaptation des attentes éducatives aux caractéristiques du groupe. Mais la gestion de l'hétérogénéité ne repose pas que sur des choix structurels posés prioritairement à l'échelle des systèmes éducatifs. L'analyse du processus d'orientation des élèves vers les classes et les écoles spéciales dans les cantons suisses, présentée dans la seconde partie de cet article, permet en effet d'illustrer combien la gestion des différences dans les systèmes éducatifs repose aussi sur des catégories cognitives mobilisées par les acteurs et sur des réalités organisationnelles affectant largement et durablement les processus d'orientation des élèves.

\section{Introduction}

Depuis longtemps déjà, la question des différences entre élèves et de leur prise en compte par l'institution scolaire occupe une place importante dans les travaux scientifiques et les débats autour de l'école.

Rappelons toutefois que la préoccupation et la valorisation de l'hétérogénéité à l'école n'est pas, loin s'en faut, consubstantielle au projet scolaire. Durant plusieurs siècles, le projet institutionnel qui donnera lieu à la forme scolaire est clairement un projet de classe, actant et perpétuant la domination de certains groupes sur d'autres. Rappelons également que pendant les premières périodes de la scolarité obligatoire et dans la plupart des pays européens (fin du $19^{\mathrm{e}}$ et début du $20^{\mathrm{e}}$ siècle), les systèmes scolaires sont clairement construits sur une logique d'ordres scolaires distincts et cloisonnés. L'ordre primaire est destiné aux enfants du peuple tandis que l'ordre secondaire (et ses écoles préparatoires) accueille les enfants de la bourgeoisie. Ce n'est que lentement, au cours du $20^{\mathrm{e}}$ 
siècle, qu'un tel cloisonnement est dénoncé et que se met progressivement en place un tronc commun d'enseignement appelé à accueillir au sein des mêmes classes et des mêmes établissements les élèves issus de différents milieux sociaux.

Mais, au-delà du principe formel d'une institution scolaire se devant d'accueillir et de former chacun, il est clair, comme l'a notamment rappelé Broadfoot (1996), que l'institution scolaire demeure tiraillée entre deux objectifs parfois contradictoires: promouvoir l'intégration de chacun et son accès à une culture commune d'une part et préparer à des positions sociales et professionnelles distinctes et hiérarchisées d'autre part. C'est à travers ce prisme et cette tension qu'il faut à nos yeux lire la question des différences et de la gestion de l'hétérogénéité dans le champ scolaire. Précisons d'emblée que le terme "hétérogénéité», s'il s'oppose à l'idée d'un traitement homogène pour tous, demeure toutefois particulièrement ambigu. Nous faisons le choix d'assumer cette ambigüité et d'en faire d'ailleurs une caractéristique de notre analyse. Dans la seconde partie du texte en particulier, notre intérêt portera sur les répertoires cognitifs de définition de l'hétérogénéité, et nous nous intéresserons aux oscillations des acteurs entre des définitions scolaires (diversité des niveaux de compétences), sociales et culturelles de l'hétérogénéité.

Cette question de la gestion des différences (terme qui, dans ce texte, sera utilisé comme un synonyme d'hétérogénéité) est une question récurrente et pour ainsi dire permanente au sein des systèmes éducatifs. Elle se pose en de multiples lieux, tant à l'échelle locale dans les classes et les établissements qu'au niveau de l'organisation et de la structure des systèmes éducatifs. Dans la première partie de ce texte, nous présentons un ensemble de résultats de recherche (relatifs aux modes de formation des classes et au recours aux filières dans l'enseignement secondaire) qui font apparaittre l'influence de formes spécifiques de gestion de l'hétérogénéité des élèves sur leurs apprentissages à l'école. C'est donc essentiellement la question des structures scolaires qui sera traitée en considérant les systèmes éducatifs comme les principales unités d'analyse. Dans la seconde partie de ce texte, nous attirerons l'attention à partir d'un exemple, le traitement des élèves à "besoins spéciaux» dans les cantons suisses, sur l'enracinement de la notion de différence et des catégories qu'elle mobilise. Nous mettrons là en évidence combien le traitement effectif des différences au sein d'un système éducatif est également ancré dans des catégories cognitives d'appréhension de la question et des catégories organisationnelles de traitement des élèves.

\section{Les modalités structurelles de gestion de l'hétérogéné $\mathrm{i}$ té}

Tous les responsables d'école et, à une autre échelle, tous les responsables d'un système éducatif sont périodiquement confrontés à cette question clé d'organisation des groupes d'élèves: faut-il rassembler au sein des classes d'abord et au 
sein des filières ensuite des élèves approximativement de même niveau scolaire? Ou faut-il au contraire, au nom d'un souci de brassage des individus (Walzer, 1977), former des groupes hétérogènes sur le plan de l'origine sociale, mais aussi des compétences scolaires, et donc retarder le plus longtemps possible le recours à des filières de formation parallèles? Cette question renvoie très clairement à des enjeux culturels, éthiques et politiques que nous n'approfondirons pas ici. Mais elle renvoie aussi à des recherches empiriques dont nous présentons ci-dessous, à partir de deux synthèses de la littérature (Dupriez, 2010; Dupriez \& Draelants, 2004), les principaux résultats.

Un argument historique (Gamoran et al., 1995) est mobilisé en faveur de la formation de classes homogènes: rassembler au sein d'une classe des élèves approximativement du même niveau scolaire permettrait au professeur de leur apporter un enseignement adapté à leur niveau, quel que soit celui-ci. Cet argument, souvent mobilisé par les enseignants, a depuis longtemps été mis à l'épreuve des faits et de nombreux travaux empiriques permettent aujourd'hui d'évaluer si, comme le suggère un tel raisonnement, les apprentissages seraient maximisés par un regroupement des élèves dans des classes homogènes.

Rappelons tout d'abord que de nombreuses recherches expérimentales ont été menées sur cette question (Slavin, 1987). Elles ont notamment mis en évidence que dans un environnement où les conditions de formation sont strictement contrôlées, et équivalentes entre les groupes, les autres élèves d'une classe n'exercent en moyenne aucune influence significative sur les apprentissages des élèves. Ces recherches expérimentales (avec distribution aléatoire des individus entre les groupes) ou quasi-expérimentales (avec des individus appariés entre les groupes) n'indiquent en général pas d'effets bénéfiques liés à l'organisation des classes de niveau ni sur l'ensemble des élèves, ni même sur certains groupes spécifiques d'élèves. Globalement, ces travaux concluent à l'absence d'un effet de pairs.

A première vue, ce résultat est difficilement compréhensible si on le met en parallèle avec les résultats de recherches semblables menées en situation réelle, dans des classes et des écoles qui ont fonctionné suivant leurs habitudes, et ouvert leurs portes aux chercheurs intéressés par l'analyse de cette problématique (Dumay, Dupriez \& Maroy, 2010; Monseur \& Crahay, 2008). De manière presque systématique, ces recherches en milieu naturel concluent à une influence du groupe d'apprentissage, laquelle correspond le plus souvent à une influence positive liée au fait d'être scolarisé dans une classe d'un haut niveau scolaire. Ces recherches mettent ainsi en évidence un effet différencié associé à la présence de classes de niveau. Les élèves les plus forts, regroupés entre eux, bénéficient d'une stimulation positive au sein du groupe. Les élèves les plus faibles, eux aussi regroupés entre eux, ne bénéficient pas de cet effet d'entrainement lié à une classe forte. Au total, les écarts entre ces deux catégories d'élèves ont dès lors tendance à s'accroître. Ces effets, positifs pour les uns et négatifs pour les autres, sont d'ampleur semblable, ce qui explique pourquoi dans la plupart des 
recherches, on n'observe pas d'effet général significatif sur l'ensemble des élèves.

Ce sont les recherches de nature plus ethnographiques qui, les premières, ont permis de comprendre la différence de résultats entre les recherches expérimentales et les recherches quantitatives menées en milieu naturel. Elles ont mis en évidence à quel point en situation naturelle, les conditions de formation peuvent varier en fonction du niveau des classes. Dans les classes les plus faibles, même si le programme d'enseignement et les objectifs sont les mêmes que dans les autres classes, le temps réel de travail est moindre, le curriculum réel est moins ambitieux, les attentes des enseignants sont moins élevées et le climat de travail est plus perturbé.

En somme, en situation naturelle, dans la mesure où le niveau de la classe covarie avec plusieurs dimensions associées à la qualité de l'enseignement, les chercheurs décèlent un avantage pour un élève à être scolarisé dans un groupe de haut niveau. À partir de tels résultats, la très grande majorité des chercheurs suggèrent un rejet des classes de niveau, car elles ne permettent pas d'augmenter le niveau moyen des systèmes éducatifs, elles ont tendance à accroître les écarts de résultats entre élèves et elles contribuent à la création de situations difficiles à gérer sur le plan socio-pédagogique, une fois que les élèves les plus faibles ont été rassemblés dans la même classe.

Au-delà de la formation des classes, la question du regroupement des élèves peut aussi être posée à d'autres échelles des systèmes éducatifs, notamment en référence aux filières telles qu'elles apparaissent généralement au cours de l'enseignement secondaire. Bien entendu, la problématique se pose ici un peu différemment dans la mesure où, inévitablement, vont se superposer dans l'analyse de l'impact des filières une influence des autres élèves scolarisés dans la même filière et un effet des différences de curriculum en fonction de la filière fréquentée.

Face à une telle problématique, un résultat est aujourd'hui stabilisé dans la littérature scientifique: le recours précoce à des filières s'accompagne d'importantes inégalités de résultats entre élèves et d'inégalités sociales de résultats plus lourdes qu'ailleurs. Cela signifie que dans les systèmes éducatifs caractérisés par le recours précoce à des filières ${ }^{1}$, on observe vers l'âge de 14-15 ans une dispersion des résultats (inégalité de résultats) plus forte qu'ailleurs ainsi qu'une influence plus forte des diplômes des parents sur le niveau des apprentissages et les parcours scolaires (inégalité sociale des résultats) (voir notamment Hanushek \& Woessmann, 2006).

Mais la recherche a également mis en évidence (voir notamment Mons, 2007; Dupriez, Dumay \& Vause, 2008) que, parmi les systèmes éducatifs qui ont un tronc commun jusque l'âge de 15 ou 16 ans et n'organisent dès lors pas de filière avant cette échéance, d'importantes différences existent quant au traitement réservé à la diversité et, en particulier, aux réponses apportées aux élèves en difficulté d'apprentissage. Mons (2007) part en effet du principe que les filières ne sont qu'une variable d'ajustement parmi d'autres que les systèmes éducatifs peuvent mobiliser afin de répondre à l'hétérogénéité des élèves. En 
dehors des filières, certains pays s'appuient fréquemment et principalement sur le redoublement comme réponse aux différences scolaires entre élèves. Ces systèmes éducatifs (en général, il s'agit des pays latins) sont ceux qui obtiennent les moins bons résultats avec les élèves faibles. Les pays anglo-saxons ont pour leur part davantage recours à des organisations de groupes de niveaux au sein des classes ou à des classes de niveau dans l'enseignement secondaire pour répondre à l'hétérogénéité du public scolaire. Et dans l'enseignement secondaire, pour la compréhension en lecture en tout cas (Mons, 2007), la présence d'importantes inégalités de résultats entre élèves dans ces pays vient confirmer les effets inégalitaires du recours aux classes de niveau. Enfin, la stratégie de réponse à l'hétérogénéité qui s'avère être la plus prometteuse, tant sur le plan de l'efficacité que de l'égalité, est celle que Mons (2007) a qualifiée de 'système d'intégration individualisée', caractérisée par une absence de filières et de redoublements, mais par le recours à la remédiation et à un accompagnement en souplesse des élèves en difficulté d'apprentissage. Cette stratégie se retrouve développée en particulier dans les pays scandinaves.

Nos propres analyses sur cette thématique ont également attiré l'attention, comme l'avaient suggéré Monseur et Demeuse (2001) et Mons (2007), sur un phénomène de vase communicant entre les variables d'ajustement: la possibilité d'orienter les élèves faibles vers l'enseignement (pré-)professionnel permet d'atténuer au début du secondaire le recours au redoublement dans les pays caractérisés par le recours précoce à des filières (Dupriez \& Dumay, 2006). Nous avons ainsi montré que dans les systèmes à filières précoces le redoublement à l'école secondaire concerne de manière moins nette et moins exclusive les élèves en difficulté d'apprentissage, probablement car une partie de ceux-ci sont orientés vers des filières moins valorisées, plutôt que de répéter une année dans l'enseignement général. C'est donc aussi la signification de ces «réponses» à l'hétérogénéité qui doit être analysée avec beaucoup de prudence. Cette signification peut varier en fonction des types de systèmes éducatifs. Ainsi, le redoublement au secondaire inférieur est clairement un indice de faiblesse scolaire dans les pays latins. Il l'est moins dans les pays germaniques qui recourent précocement aux filières et qui gèrent à travers celles-ci l'essentiel des différences de performances scolaires. Entre pays à la structure proche, la signification d'une variable peut aussi varier. À ce titre, les filières professionnalisantes semblent davantage faire office en Belgique francophone de filières de relégation scolaire et sociale qu'en Allemagne (ou en Autriche) où, sur la base des données de PISA 2003 (Dupriez $\&$ Dumay, 2006), il apparaît que ces filières attirent également des élèves de très bon niveau scolaire.

La synthèse présentée ci-dessus a permis de mettre en évidence des résultats de recherche assis sur de solides bases empiriques et faisant presque l'objet d'un consensus au sein de la communauté scientifique. Deux critiques au moins doivent toutefois être adressées à ces travaux et serviront de fil conducteur à la seconde partie de ce texte. D'une part, une modalité structurelle de gestion de l'hétéro- 
généité des élèves n'est pas prise en compte dans les travaux présentés jusqu'à présent, c'est l'orientation (là où elle existe) vers l'enseignement spécialisé. Il s'agit pourtant dans certains pays d'un mécanisme clé de gestion des trajectoires scolaires et de sélection précoce des élèves. D’autre part, les données présentées jusqu'à présent attirent essentiellement l'attention sur des choix structurels posés à l'échelle d'un système éducatif. Il serait pourtant naïf de croire qu'il suffirait de poser les bons choix structurels pour faire évoluer un système éducatif dans la direction souhaitée. L'analyse de l'orientation vers l'enseignement spécialisé en Suisse, présentée ci-dessous, va nous permettre de traiter plus en détails cette double critique et de faire apparaitre en même temps l'importance des dimensions organisationnelles et cognitives liées à cette question.

\section{Une étude de cas: L'orientation vers les classes et les écoles spéciales dans les cantons suisses}

La seconde partie de ce texte s'appuie sur une recherche doctorale menée par l'un des auteurs (Shaik, 2011). Après une brève présentation du contexte institutionnel suisse et, en particulier, des voies d'orientation vers les classes et les écoles "spéciales», le texte met en évidence une série de travaux qui ont veillé à comprendre pourquoi les élèves issus de l'immigration sont à ce point surreprésentés dans l'enseignement spécialisé, et, dans la suite de leur parcours scolaire, peu présents dans les filières de formation les plus valorisées (Kronig, 2000). Le texte vise à faire ressortir l'imprégnation des acteurs et de leurs décisions d'orientation des élèves dans des schèmes cognitifs et dans des environnements organisationnels plus ou moins propices à l'externalisation des problèmes éducatifs vers une structure spécifique, telle que l'enseignement spécialisé.

\section{L'environnement structurel}

En Suisse à l'école primaire, les élèves identifiés comme ayant des difficultés d'apprentissage ou présentant un handicap peuvent être pris en charge au sein d'une classe ordinaire, où ils côtoient alors d'autres enfants au parcours scolaire «classique». Mais, en fonction du système en vigueur dans chaque canton, ils peuvent aussi être orientés vers des classes spéciales (Sonderklassen), parallèles à la classe ordinaire - et alors situées dans la même école - ou enfin dans des écoles spéciales (Eckhart et al., 2011; Lischer, 1997). Le processus d'orientation vers une classe ou une école spéciale est généralement initié par l'enseignant titulaire d'une classe, qui peut en référer, selon les cantons, à un psychologue scolaire, en réponse à des insuffisances ou à des difficultés de l'enfant. Sur la base d'un examen par le psychologue, un rapport est envoyé au conseil scolaire local. La décision officielle d'orientation appartient alors aux membres de ce conseil et non aux parents (Lanfranchi, 2002). 
Eu égard toutefois à l'organisation scolaire suisse très décentralisée, il existe autant de systèmes scolaires que de cantons, et des variations significatives dans ce processus existent entre les cantons. Le taux d'orientation des élèves immigrés vers les classes spéciales differe considérablement d'un canton à l'autre, et contrairement à certains stéréotypes, ces variations ne correspondent pas aux taux de population immigrée observés dans les cantons (Lischer, 2003). Il semble donc que, pour cette catégorie d'élèves, la décision d'orientation soit significativement influencée par des décisions préalables concernant d'autres élèves immigrés.

À l'échelle nationale, Kronig et al. (2000) constatent par ailleurs que ces orientations ont plus que triplé entre 1980 et 2000, alors que la présence de ces enfants dans les classes ordinaires n'a augmenté sur cette période que de $35 \%$. Plusieurs recherches attirent par ailleurs l'attention sur le fait que cette orientation vers l'enseignement spécialisé n'est pas toujours justifiée au regard des compétences objectives des élèves et que les progrès des élèves en difficulté d'apprentissage sont plus grands s'ils demeurent intégrés dans l'enseignement ordinaire que s'ils sont scolarisés dans une classe spéciale (Eckhart et al., 2011).

Ces données rapidement résumées ici font ainsi apparaître la très nette surreprésentation des jeunes issus de l'immigration dans les classes et les écoles spécialisées, lesquelles apparaissent bien comme une autre variable d'ajustement des systèmes éducatifs face à l'hétérogénéité des élèves, et attirent par ailleurs notre attention sur un processus d'orientation qui semble varier significativement en fonction des cantons.

\section{Les modalités cognitives et organisationnelles d'appréhension des élèves en difficulté et de leur orientation}

Plusieurs recherches menées au cours de ces dix dernières années ont permis de mieux comprendre les processus qui président à l'orientation des élèves vers l'enseignement spécialisé. Nous allons en rendre compte ci-dessous dans la mesure où elles révèlent, non seulement des éléments caractéristiques de la situation suisse mais aussi, plus globalement, des freins à tout processus de changement des modes de gestion de l'hétérogénéité. Dans l'étude WASA ${ }^{2}$ sur les processus d'orientation des élèves vers l'enseignement spécialisé, les chercheurs (Lanfranchi et al., 2005) ont mené une enquête auprès de 655 enseignants et 207 psychologues scolaires dans onze cantons suisses germanophones (Lanfranchi, 2005; Lanfranchi \& Jenny, 2005; Schüpbach, 2004). Cette recherche WASA a ensuite été répliquée dans les cantons de Suisse romande à travers les travaux de Baumberger et al. $(2007,2009)$ conduisant à des résultats similaires.

A partir d'études de cas détaillées de situations scolaires où le nom de l'enfant, la profession et l'origine du père étaient modifiées, les enseignants interrogés devaient indiquer les mesures qu'ils prendraient face aux difficultés rencontrées. Un résultat majeur de cette recherche est le constat que les réponses des enseignants sont fortement influencées par le niveau socio-économique de l'élève et 
par son éventuel statut d'immigré. Les psychologues scolaires pour leur part n'ont pas été influencés par l'origine ethnique des parents, mais bien par le statut social des élèves.

De toute évidence, le jugement de ces professionnels est entaché de stéréotypes caractérisant certaines catégories d'élèves. Interrogés sur leurs pratiques d'orientation et sur le pourcentage élevé d'élèves immigrés présents dans l'enseignement spécialisé, de nombreux enseignants évoquent à ce propos des explications telles que les difficultés langagières, la distance culturelle, le faible niveau d'éducation des parents, la difficulté à s'adapter au système scolaire local (Bannwart \& Bättig, 2002; Shaik, 2011). Dans une telle perspective, les difficultés d'apprentissage de ces élèves semblent être intrinsèquement liées à leur position sociale et culturelle, et par ailleurs presque attendues et prévues par les enseignants.

En outre, les résultats de l'étude WASA montrent une importante variabilité des réponses relatives à l'orientation des élèves d'un canton à l'autre (Lanfranchi, 2005; Baumberger et al., 2007, 2009). Alors que dans certains petits cantons, les enseignants ont tendance à imaginer des solutions internes à l'école, parfois à travers des formes innovantes d'intégration, les enseignants travaillant dans les cantons les plus importants ont davantage tendance à déléguer le problème auprès d'un service psychologique externe à l'école. Enfin, au-delà d'une interpellation des psychologues et des enseignants sur la réponse à apporter aux difficultés des enfants, la recherche WASA (Lanfranchi et al., 2005) demandait également aux professionnels si le cas présenté devait être compris comme une «faiblesse» révélatrice d'un retard dans le développement ("Entwicklungskrise») ou comme une "perturbation" plus fondamentale. À nouveau, la relation entre la perception des enseignants et les structures organisationnelles en place est ici manifeste: dans les écoles où des logopédistes sont présents et où des élèves en difficulté sont intégrés dans les classes ordinaires, l'attribution de "faiblesse» est la plus fréquente et les enseignants suggèrent plutôt un maintien dans la classe, éventuellement avec l'aide d'un enseignant expérimenté. Dans les autres environnements scolaires, l'attribution de "perturbation" est la plus fréquente, et s'accompagne souvent d'une proposition d'orientation vers une classe spéciale.

Gomolla \& Radtke (2002), à partir d'une recherche menée en Allemagne mettent également en relation les décisions d'orientation avec les structures organisationnelles en présence. Plus précisément, ils montrent que tant qu'un certain nombre de places dans l'enseignement spécialisé sont disponibles ou planifiées, elles seront presque toujours utilisées, en dépit de tous les autres efforts visant à réduire de telles orientations.

Hansen (2012) parle à cet égard de «l'identité professionnelle» des enseignants (professional self-concept), construite à partir de leur compréhension des situations professionnelles, des catégories discursives mobilisées et du sens qu'ils donnent aux situations. En fonction de ces grilles d'interprétation, les enseignants positionnent les élèves entre l'inclusion et l'exclusion. Dans une telle dynamique, les acteurs scolaires tendent à mobiliser des routines cognitives qui 
simultanément dédouanent l'établissement scolaire de toute responsabilité face aux difficultés rencontrées et projettent l'origine de ces difficultés sur l'enfant et sa famille. Les dimensions ethniques ou culturelles sont alors mobilisées comme dimensions déterminantes permettant à la fois de nommer et d'expliquer les difficultés. Le dernier élément d'un tel «répertoire cognitif» consiste à solliciter la structure scolaire conçue pour répondre à de telles difficultés, c'est-à-dire l'enseignement spécialisé.

Pour illustrer cette problématique, nous nous baserons sur des données récoltées au cours d'une recherche doctorale. Celle-ci consistait en une analyse des discours politiques relatifs à l'intégration (Shaik, 2011). L'approche tant théorique que méthodologique de cette recherche visait à comprendre les processus politiques en tant que discours (Fairclough, 2009; Halliday \& Hasan, 1985; cité par Luke, 1996, p. 14). À travers ce prisme de l'analyse discursive, il s'agissait d'observer et analyser non seulement (a) des textes et documents politiques, mais aussi (b) les réseaux locaux de décideurs politiques et les acteurs intermédiaires (Grek et al., 2009). La réalisation de 21 entretiens semi-directifs avec de tels acteurs a permis un examen approfondi de la compréhension qu'ont les acteurs des concepts ou catégories de "différence» et d' "hétérogénéité», ainsi que leur contribution à l'intégration de ces notions dans les discours politiques. Enfin, il s'agissait également d'étudier (c) la manière dont ces acteurs se réferent aux politiques en mobilisant des connaissances particulières (Arnott \& Ozga, 2010).

\section{L'auto-alimentation et la croissance de l'enseignement spécialisé}

Dans les récits issus des entretiens menés en 2008/2009 pour cette recherche (Shaik, 2011), des acteurs du canton de Zurich soulignent ce qu'ils voient comme mécanisme d'auto-alimentation de l'orientation des élèves et explicitent les conditions à la fois cognitives et organisationnelles d'un tel processus. Nous en reprenons ici deux extraits notoires:

J'étais là de 84 à 94, quand il y avait quelque chose comme une 'éducationalisation des besoins spéciaux des enfants'. Des classes spéciales ont poussé comme des champignons. L'idée était d'optimiser le parcours éducatif de chaque enfant; il fallait trouver un expert pour chaque difficulté. [...] Je suis parti car je ne voulais plus représenter ce tri des enfants. En dix ans, j'ai placés 1500 enfants chaque année, en partie contre la volonté de la famille et contre ma propre conviction. Jusqu'à l'année 2000, cette éducationalisation des besoins spéciaux a augmenté. [...]

$\mathrm{Au}$ début, on pensait que c'était une réussite, que nous avions nommé des spécialistes et qu'ils travailleraient mieux que les généralistes. [...] Nous sommes nous-mêmes responsables de cela, parce qu'on a vu un progrès dans ce dispositif; il est venu comme une sorte de pensée clinique. Et puis, à mon avis à un certain moment, nous n'avons plus 
pris conscience de cette ampleur et on ne voyait plus ce qui se passait.

(Extrait d'entretien avec un ancien psychologue scolaire)

Un second entretien attire l'attention sur la dimension historique d'un tel processus et sur la manière dont les catégories cognitives s'inscrivent dans des organisations et des institutions solidement implantées dans le pays:

Le problème est que les 'éducateurs spécialisés' considèrent les immigrés comme leur domaine. En Suisse, nous avons eu la première chaire de pédagogie thérapeutique en Europe en 1931; la première institution de formation d'enseignants spécialisés en pédagogie thérapeutique. Depuis lors, nous avons une croissance massive des institutions liées aux 'besoins spéciaux', du personnel et de l'argent massivement orientés vers ce secteur [...] Nous avons un concordat scolaire ordinaire et un concordat pour les 'besoins spéciaux'; deux systèmes scolaires distincts, qui se sont développés l'un à coté de l'autre, depuis une centaine d'années. Le système scolaire ordinaire renvoie des enfants vers le système d'enseignement spécialisé, lorsque les enseignants estiment qu'ils ne sont pas responsables pour des enfants ne correspondant pas à leurs repères normatifs. Les enfants immigrés ont effectivement été traités de cette manière. Ils ne correspondent pas à la normalité de la classe moyenne suisse, et ont donc été confiés aux pédagogues de l'enseignement spécialisé. (Extrait d'entretien avec un membre de la Conférence des Recteurs des Universités suisses)

Ces exemples de discours narratifs font apparaître également une autocritique des pratiques de séparation et soulignent le caractère historique du traitement des «besoins spéciaux» de même que la manière dont les catégories cognitives des acteurs (les termes même de "besoins spéciaux» ou de "pédagogie thérapeutique», par exemple) sont adossées à des structures organisationnelles. Une ambigüité se présente dans les discours des acteurs liés au champ scolaire à travers tous les entretiens menés; ils souhaitent simultanément s'impliquer en faveur de l'intégration des enfants avec des «besoins spéciaux», tout en souhaitant préserver le système d'enseignement spécialisé.

\section{Les catégories cognitives liées aux aspects "culturels ou ethniques» entendus comme «besoins spécifiques»}

Comme le souligne l'extrait d'entretien repris ci-dessous, le rapport entre le concept d'intégration et les politiques migratoires suisses peut avoir favorisé la mobilisation des catégories cognitives "culturelles ou ethniques» entendues comme «besoins spécifiques».

Quand on parlait d'intégration, on visait l'intégration des enfants handicapés et donc l'enseignement spécialisé. Depuis peu, le débat autour de l'intégration dans le champ scolaire s'est articulé autour de la notion d'inclusion. Parallèlement, les champs de la migration et de l'ensei- 
gnement spécialisé ont repris cette question en même temps. Mais l'enseignement spécialisé a une tradition plus ancienne et, dans cette optique, on doit toujours clarifier les différences entre les discours sur la migration et ceux sur l'enseignement spécialisé, parce qu'il y a des chevauchements. Concernant les classes spéciales [avec des enfants présentant des besoins spécifiques], on observe une trop grande ségrégation de tous les enfants handicapés. Certains se demandent s'il n'y a pas une surreprésentation des migrants dans ces classes. Ne sont-ils pas classifiés comme présentant des besoins spécifiques en raison de leur comportement culturel?

(Entretien réalisé avec un acteur de la Conférence suisse des directeurs cantonaux de l'instruction publique - CDIP).

Cet extrait souligne la polysémie de la notion d'intégration, qui puise simultanément ses racines dans les discours migratoires et dans le champ de l'enseignement spécialisé. Cette polysémie est propice à un traitement ambigu de la culture et des élèves issus de l'immigration, la catégorisation culturelle étant susceptible d'être associée à l'idée d'un «handicap». Ce type de catégorisation aurait, selon certains, favorisé une surreprésentation des élèves issus de la migration dans les classes spéciales. En outre, rappelons que la ségrégation est intimement liée à l'idéologie de spécialisation mentionnée précédemment.

\section{L'usage des données PISA comme mode d'identification des élèves en difficulté}

Par ailleurs, la saillance de «l'origine ethnique» des élèves comme un aspect de la différenciation dans l'enseignement est évidemment un point très délicat dans le contexte suisse, avec en toile de fond un débat très polémique autour de l'immigration, de "la culture suisse» et de la référence à l'«identité nationale». Des études internationales comme PISA ont rendu particulièrement visible le niveau académique faible des étudiants migrés dans les écoles suisses. Mais, dans l'espace politique et médiatique, le débat reste ouvert autour de l'interprétation des faibles performances d'une partie de la population scolaire, et des inégalités massives entre différents types d'élèves. À nouveau, en termes de catégories cognitives, le cœur du débat est dans la manière dont il faut nommer les groupes d'élèves dont les performances varient.

La question de l'inégalité scolaire était fort discutée dans les années septante, puis elle a disparu, et avec PISA, est réapparue massivement. Elle est, je dirais, une des questions les plus urgentes des politiques d'éducation. Et maintenant, bien sûr, elle est fortement associée à la question de la migration. En fin de compte, l'enquête PISA montre qu'on ne peut pas parler d'égalité des chances pour les enfants issus de milieux socio-économiquement défavorisés. Et l'aspect «issus de migration» ou «langue étrangère» est moins important. Mais il y a souvent des chevauchements; souvent, les migrés viennent de statuts socio-économiques inférieurs. Mais 
en réalité, la classe sociale est toujours le facteur déterminant.

(Extrait d'entretien avec un acteur de la Direction de l'Éducation et le Bureau des écoles publiques du canton de Zürich)

Oui, il y a l'idée de comparer l'égalité des chances; comparer le groupe qu'on veut intégrer avec la population locale. Ensuite, on peut établir s'il existe des déficits. ... ça déclenche la question quels sont les groupes à comparer. On ne peut pas simplement comparer l'ensemble de la population suisse, dans laquelle il y a les différentes classes sociales, avec un groupe de migrés. [...] On peut dire 'comparer les même avec les mêmes'. Des Suisses de la même classe sociale, mais comment peut-on définir cela? Mesurer le contexte socio-économique est effectivement aussi un problème de données, car on ne peut pas mesurer cela en Suisse. [...] Ici, il s'agit seulement de la comparaison suisse-étranger, qui est tout ce qu'on peut récupérer. Le niveau de scolarité d'une personne, son emploi, son activité actuelle, ce n'est dans aucun registre; il doit donc y avoir d'autres enquêtes ou sources.

(Extrait d'entretien avec un expert statistique de l'Administration fédérale).

La référence à l'immigration, largement présente quand il s'agit de nommer les élèves avec des "besoins spéciaux», semble ainsi masquer un point politiquement sensible, dont l'interprétation oscille entre une question de politique d'intégration des populations immigrées et une autre question plus large, d'inégalité sociale d'accès à l'éducation dans le pays.

\section{Conclusion}

La première partie de cet article nous a permis de mettre en évidence les résultats de recherches quantitatives montrant les implications sur les parcours scolaires des élèves de différents modes de gestion de l'hétérogénéité des niveaux scolaires des élèves. Il en ressort que les diverses formes d'isolement des élèves les plus faibles conduisent presque systématiquement à un accroissement des écarts avec les autres, vu la tendance volontaire (curriculum formel) ou involontaire (curriculum réel) à adapter les objectifs et les contenus d'enseignement aux caractéristiques des groupes-classes. Ces recherches mettent également en évidence la tendance des filières organisées précocement à accroître les inégalités de résultats entre élèves et à exacerber la relation entre l'origine sociale et les performances scolaires.

La seconde partie de ce texte a présenté brièvement le résultat d'une analyse $\mathrm{du}$ processus d'orientation des élèves (notamment issus de l'immigration) vers les classes spéciales en Suisse. Cette étude de cas est un complément original aux travaux cités ci-dessus. D'une part, car elle nous permet de mettre en évidence l'importance d'une modalité de gestion de l'hétérogénéité (l'orientation vers l'enseignement spécialisé) non prise en compte dans les travaux cités en amont. 
D'autre part et surtout, car cette analyse fine des paramètres qui participent aux prises de décision des acteurs (les enseignants et les psychologues scolaires en particulier) permet de mettre en évidence la complexité des processus de prise de décision, et la manière dont ils sont ancrés dans un environnement à la fois culturel (des schèmes cognitifs) et organisationnel historiquement constitué.

D’un point de vue cognitif, les éléments présentés ci-dessus font ressortir un certain nombre de stéréotypes qui participent à la catégorisation des élèves présentant des difficultés d'apprentissage. Les travaux de Lanfranchi (2005) et de ses collègues ont ainsi mis en évidence la propension des enseignants à orienter plus vite vers l'enseignement spécialisé les élèves issus de l'immigration et de milieux socioculturels peu favorisés. Une telle propension est souvent justifiée par les enseignants à travers un schème articulant des déficits intrinsèques de ces élèves (maîtrise de la langue d'enseignement, distance culturelle ...), l'absence de responsabilité des écoles dans le traitement de ces difficultés et la présence des classes et écoles spéciales comme lieu organisationnellement constitué pour traiter ces difficultés, à extraire de l'espace scolaire ordinaire. Les extraits d'entretien présentés ci-dessus attirent également l'attention sur le fait qu'un tel mode de pensée n'est pas une production isolée d'un individu, mais émerge comme une pensée cohérente dans un système éducatif qui a organisé un traitement parallèle des besoins particuliers dans des entités spécifiques. Notre analyse fait également ressortir l'ambigüité de la catégorisation des élèves «à risque», laquelle oscille entre une définition ethnique et une définition socioéconomique. À cet égard, un des intérêts de l'enquête PISA est d'avoir mis en évidence que les mauvaises performances scolaires en Suisse sont davantage associées au niveau socioéconomique des élèves qu'à leur lieu de naissance ou à la nationalité de leurs parents. Cette tension apparaît également dans les discours des acteurs impliqués dans la traduction des politiques éducatives, lesquels oscillent entre une approche de l'intégration scolaire en tant que politique migratoire et une approche plutôt inclusive, fondée sur une problématique d'apprentissage et d'inégalités scolaires.

D'un point de vue organisationnel, il ressort de ces travaux que la propension à orienter des élèves vers les classes ou les écoles spéciales varie en fonction des cantons. Elle varie également en fonction de paramètres organisationnels très spécifiques: la tendance à avoir au préalable orienté un grand nombre d'élèves vers ces structures et la facilité à réaliser une telle orientation (la disponibilité des places dans l'enseignement spécialisé, par exemple) tend à accroître les taux d'orientation. Au contraire, là où des expériences d'intégration ont eu lieu, les enseignants ont moins tendance à externaliser les élèves en difficulté en demandant qu'ils quittent leur classe d'origine.

En somme, ces recherches font apparaître que le processus global de gestion d'élèves nécessairement différents, s'il repose sur certains choix structurels majeurs (tel que l'âge d'apparition des filières dans un système éducatif), repose aussi sur des caractéristiques plus locales telles que la présence ou l'absence d'alternatives à proximité et sur des schèmes cognitifs (souvent historiquement 
et collectivement construits) plus ou moins propices à une prise en compte de la différence comme une caractéristique inhérente à tout processus éducatif. Tout processus de changement initié dans un système éducatif devra être autant attentif à ces dimensions qu'à la définition des paramètres structurels souhaités.

\section{Notes}

1 Les pays qui occupent la position la plus extrême sur ce continuum sont l'Allemagne (dans la plupart des Länder, les filières sont organisées dès à l'âge de 10 ans) et l'Autriche (11 ans).

2 Wachstum des Sonderpaedagogischen Angebots.

\section{Références}

Arnott, M. \& Ozga, J. (2010). Education and Nationalism: The discourse of education policy in Scotland. Discourse, 31 (4), 335-351.

Bannwart, B. \& Bättig, J. (2002). Migrantenkinder in Klein- und Sonderklassen: Ursachen und lösungsorientierte Ansätze. Dissertation, Interkantonale Hochschule für Heilpädagogik Zürich.

Baumberger, B., Lischer, R., Moulin, J.-P., Doudin, P.-A. \& Martin, D. (2007). Intégration ou séparation des élèves étrangers: comparaison entre réalité et perceptions. Pédagogie spécialisée, 3, 27-32.

Baumberger, B., Doudin, P.-A. \& Martin, D. (2009). Études de l'usage des mesures de pédagogie spécialisée en Suisse latine. Formation et pratiques d'enseignement en questions, 9, $117-134$.

Broadfoot, P. (1996). Education, assessment and society: A sociological analysis. Buckingham: Open University Press.

Dumay, X., Dupriez, V. \& Maroy, C. (2010). Ségrégation entre écoles, effets de la composition scolaire et inégalités de résultats. Revue française de sociologie, 51 (3), 461-480.

Dupriez, V. (2010). Séparer pour réussir? Les modalités de groupement des élèves (Coll. Principes de la planification en éducation, $\mathrm{N}^{\circ}$ 93). Paris: UNESCO.

Dupriez, V. \& Draelants, H. (2004). Classes homogènes versus classes hétérogènes: les apports de la recherche à l'analyse de la problématique (Note de synthèse). Revue française de pédagogie, 148, 145-165.

Dupriez, V. \& Dumay, X. (2006). Élèves en difficulté d'apprentissage: parcours et environnements éducatifs différenciés en fonction des structures scolaires. Les Cahiers de recherche en éducation et en formation, 51, 3-22.

Dupriez, V., \& Vause, A. (2008). How do school systems manage pupils' heterogeneity? A reanalysis of PISA 2003. Comparative Education Review, 52 (2), 245?273.

Eckhart, M., Haeberlin, U. \& Sahli Lozano, C. (2011). Langzeitwirkungen der schulischen Integration. Bern: Haupt Verlag.

Fairclough, N. (2009). A dialectical-relational approach to critical discourse analysis in social research. In R. Wodak \& M. Meyer (Eds.), Methods for Critical Discourse Analysis (pp. 162-187). London: Sage.

Gamoran, A., Nystrand, M., Berends, M. \& LePore, P. (1995). An organizational analysis of the effects of ability grouping. American Educational Research Journal, 32 (4), 687-715.

Gomolla, M. \& Radtke, F.-O. (2002). Institutionelle Diskriminierung: Die Herstellung ethnischer Differenz in der Schule. Opladen: Leske \& Budrich.

Grek, S., Lawn, M., Lingard, B., Ozga, J., Rinne, R., Segerholm, C. \& Simola, H. (2009). National policy brokering and the construction of the European Education Space in England, Sweden, Finland and Scotland. Comparative Education, 45 (1), 5-21. 
Hanushek, E. \& Woessmann, L. (2006). Does educational tracking affect performance and inequality? Differences-in-differences evidence across countries. Economic Journal, 116, C63-C76.

Hansen, J. H. (2012). Limits to inclusion. International Journal of Inclusive Education, 16 (1), 89-98.

Kronig, W., Haeberlin, U. \& Eckhart, M. (2000). Immigrantenkinder und schulische Selektion: Pädagogische Visionen, theoretische Erklärungen und empirische Untersuchungen zur Wirkung integrierender und separierender Schulformen in den Grundschuljahren. Bern: Haupt.

Lischer, E. (2003) Integrierte Fremde? - Eine statistische Antwort. Auslaendische Kinder und Jugendliche im schweizeri schen Bildungssystem. In: Schweizerische Konferenz der nationalen Erziehungsdirektoren (EDK) (Hrsg.): Schul- und Bildungslaufbahn von immigrierten "leistungsschwachen» Schülerinnen und Schülern. Schlussbericht. CONVEGNO 2002. Bern 2003, 11-21.

Lanfranchi, A. (2002). Schulerfolg von Migrationskindern. Die Bedeutung familienergänzende Betreuung im Vorschulalter. Opladen: Leske + Budrich.

Lanfranchi, A. (2005) Nomen est Omen: Diskriminierung bei sonderpädagogischen Zuweisungen. Schweizerische Zeitschrift für Heilpädagogik, 7/8, 45-48.

Lanfranchi, A. \& Jenny, G. (2005). Prozesse der Zuweisung von Kindern mit Problemen zu sonderpädagogischen Massnahmen. In K. Häfeli \& P. Walther-Müller (Eds.), Das Wachstum des sonderpädagogischen Angebots im interkantonalen Vergleich (pp. 217-278). Luzern: Schweizerische Zentralstelle für Heilpädagogik.

Luke, A. (1996), «Text and Discourse in Education: An Introduction to Critical Discourse Analysis.» Review of Research in Education, 21: 3-48.

Marks, G. N. (2005). Accounting for immigrant non-immigrant differences in reading and mathematics in twenty countries. Ethnic and Racial Studies, 28 (5), 925-946.

Mons, N. (2007). Les nouvelles politiques éducatives: la France fait-elle les bons choix? Paris: PUF.

Monseur, C. \& Crahay, M. (2008). Composition académique et sociale des établissements, efficacité et inégalités scolaires: une comparaison internationale. Revue française de pédagogie, 164, 55-65.

Monseur, C. \& Demeuse, M. (2001). Gérer l'hétérogénéité des élèves: méthodes de groupement des élèves dans l'enseignement obligatoire. Cahiers du service de pédagogie expérimentale, 7-8, 25-51.

Schüpbach, E. (2004). Das Wachstum der sonderpädagogischen Massnahmen in der Schule. Eine empirische Untersuchung der Zuweisungsprozesse in die Sonderschule (Unveröff. Lizentiatsarbeit). Zürich: Universität Zürich, Institut für Sonderpädagogik.

Shaik, F. (2011) Education, Governance and Frames of Political Membership: Migrant 'Integration' Policy as Discourse in the Swiss case within Europe (PhD Thesis). Edinburgh: University of Edinburgh.

Slavin, R. E. (1987). Ability grouping and student achievement in elementary schools: A best evidence synthesis. Review of Educational Research, 57 (3), 293-336.

Walzer, M. (1997). Sphères de justice. Paris: Seuil.

Mots-clés: Hétérogénéité, inégalités, orientation, ségrégation, systèmes éducatifs 


\section{Die strukturellen, organisationalen und kognitiven Herausforderungen im Umgang mit der Diversität von Schülerinnen und Schülern in Bildungssystemen}

\section{Zusammenfassung}

Dieser Artikel befasst sich mit unterschiedlichen Möglichkeiten, die Heterogenität der Schülerschaft in Bildungssystemen zu identifizieren und mit dieser umzugehen. Anhand von gut etablierten Forschungsergebnissen wird gezeigt, dass durch separierende Formen wie z.B. Sonderklassen, Niveau-Kurse und optionale Angebote die Unterschiede zwischen benachteiligten Schülerinnen und Schülern und den anderen fast immer zunehmen. Grund dafür ist, dass die pädagogischen Erwartungen an die Charakteristik der jeweiligen Gruppe angepasst werden. Der Umgang mit schulischer Heterogenität ist nicht nur auf strukturelle Entscheidungen auf der Systemebene beschränkt. Der zweite Teil dieses Artikels zeigt anhand einer Analyse der Zuweisung von Schülerinnen und Schülern in Sonderklassen und -schulen in den Schweizer Kantonen, wie Unterschiede in der Verwaltung der Bildungssysteme, die nebst organisatorischen Gegebenheiten auch kognitive Kategorien der Akteure betreffen, umfassend und langfristig die Bildungslaufbahn von Schülerinnen und Schülern beeinflussen.

Schlagworte: Heterogenität, Ungleichheiten, Orientierung, Segregation, Bildungssysteme

\section{Le sfide strutturali, organizzative e cognitive nella gestione della diversità degli studenti nei sistemi educativi.}

\section{Riassunto}

Questo articolo di concentra sulle diverse modalità di identificare e affrontare la sfida dell'eterogeneità degli studenti nei sistemi educativi. Vengono raccolti diversi risultati di ricerche riconosciute che illustrano come il raggruppamento di studenti vulnerabili in gruppi particolari (classi, percorsi, livelli, opzioni, ecc.) si accompagna sistematicamente con l'aumento delle differenze tra questi allievi e gli altri, in particolare attraverso un processo di adattamento delle aspettative educative alle caratteristiche del gruppo. L'analisi del processo di orientamento degli allievi verso le classi e le scuole speciali nei cantoni svizzeri, presentata nella seconda parte dell'articolo, permette di illustrare come la gestione delle differenze nei sistemi educativi si fonda anche su categorie cognitive mobilitate dagli attori e su realtà organizzazionali che hanno un effetto profondo, continuo e duraturo sui processi di orientamento degli allievi.

Parole chiave: Eterogeneità, disuguaglianza, orientamento, segregazione, sistemi educativi 


\section{The structural, organizational and cognitive challenges of student diversity management in education systems}

\section{Abstract}

This paper focuses on different ways of identifying and addressing the issue of student heterogeneity in educational systems. It convenes well-established research results that illustrate how the institutional regrouping of vulnerable students in specific or special groups (whether in classes, streams, tracks, options, etc.) almost always is accompanied by an increase in the differences between these particular students and others, notably through a process of alignment of educational expectations to the features of the group. But the management of pupil heterogeneity does not solely rely on structural choices made primarily at the level of educational systems. The analysis of orientation processes of students toward special education classes and schools in the Swiss cantons, presented in the second part of this paper, makes it possible to illustrate how differences in the management of education systems is also based on cognitive categories mobilized by actors and on organizational realities, which then affect orientation processes of students in a significant and continuous manner.

Key words: Heterogeneity, inequalities, orientation, segregation, educational systems 
